Pacific Journal of Mathematics

THE STRUCTURE OF A SPECIAL CLASS OF WEIGHTED 


\title{
THE STRUCTURE OF A SPECIAL CLASS OF WEIGHTED TRANSLATION SEMIGROUPS
}

\author{
Mary R. Embry and Alan Lambert
}

A special class of weighted translation semigroups $\left\{S_{t}\right\}$ on $\mathscr{L}^{2}\left(\mathscr{R}_{+}\right)$is studied. The weakly closed algebra $\mathscr{A}$ generated by the semigroup is maximal abelian and the spectra of elements of $\mathscr{A}$ are studied. It is shown that each densely defined linear transformation commuting with $\mathscr{A}$ is closable and that every transitive algebra containing $\mathscr{A}$ is weakly dense in the full algebra of operators on $L^{2}\left(\mathscr{R}_{+}\right)$.

1. Introduction. A weighted translation semigroup $\left\{S_{t}\right\}$ with symbol $\phi$ is defined on $L^{2}\left(\mathscr{R}_{+}\right)$by

$$
\left(S_{f} f\right)(x)= \begin{cases}\frac{\phi(x)}{\phi(x-t)} f(x-t) & \text { for } 0 \leqq t \leqq x \\ 0 & \text { for } t>x\end{cases}
$$

where $\phi$ is a continuous, complex-valued function on $\mathscr{R}_{+}$such that $\phi(x) \neq 0$ for $x$ in $\mathscr{R}_{+}$. These semigroups were studied in [2] and [3]. In [3] strongly continuous subnormal weighted translation semigroups are characterized as those for which $\phi^{2}$ is a Laplace-Stieltjes Transform of a probability measure. In [4] a more general type of weighted translation semigroup is studied.

To insure the strong continuity of $\left\{S_{t}\right\}$ we assume that $\sup _{x \in \Re_{+}}|\phi(x+t) / \phi(x)| \leqq M e^{w t}$ for all $t$ and some constants $M$ and $w$ [2, Lemma 2.1]. Two weighted translation semigroups with symbols $\phi$ and $\rho$ are unitarily equivalent if and only if $|\phi / \rho|$ is constant [2, Theorem 2.5]. Thus without loss of generality we assume that $\phi$ is positivevalued and that $\phi(0)=1$.

Throughout the paper unless otherwise indicated we shall assume further that $\int_{0}^{x}(\phi(x) / \phi(t) \phi(x-t))^{2} d t$ is bounded and shall say that $\phi$ is of bounded kernel type. For such a $\phi$ and for each $f$ in $L^{2}\left(\mathscr{R}_{+}\right)$we define

$$
A_{f}=\int_{0}^{\infty} \frac{f(t)}{\phi(t)} S_{t} d t
$$


In $\S 2$ we show that $\left\{A_{f}: f \in L^{2}\left(\mathscr{R}_{+}\right)\right\}$is a subalgebra of $B\left(L^{2}\right)$, the full algebra of operators on $L^{2}\left(\mathscr{R}_{+}\right)$. We denote $\left\{A_{f}: f \in L^{2}\left(\mathscr{R}_{+}\right)\right\}$by $\mathscr{A}_{0}$ and its closure in the weak operator topology by $\mathscr{A}$. In Theorem 2.6 we show that $\mathscr{A}$ is a maximal abelian algebra and that $\mathscr{A}_{0}$ is a proper ideal of $\mathscr{A}$.

In $\$ 3$ we establish a basic relation between the multiplicative linear functionals on $\mathscr{A}$ and the elements of $L^{2}\left(\mathscr{R}_{+}\right)$of the form $e^{\lambda t} / \phi(t)$. This relation enables us in Theorem 3.5 to determine completely the spectrum of each element of $\mathscr{A}_{0}$. In $\$ 4$ it is shown that any densely defined linear transformation commuting with $\mathscr{A}$ is closable. This result enables us to apply Arveson's Density Theorem to show that if $e^{\lambda z} / \phi(t) \in L^{2}\left(\mathscr{R}_{+}\right)$for some $\lambda$, then any transitive subalgebra of $B\left(L^{2}\right)$ which contains $\mathscr{A}$ is weakly dense in $B\left(L^{2}\right)$. Finally in $\$ 5$ certain function theoretic considerations related to $\phi$ are investigated. It is shown in Corollary 5.5 that if the associated semigroup is hyponormal then $\phi$ is not of bounded kernel type.

Throughout the paper the following notation is used: $H=$ $\left\{\lambda: e^{\lambda t} / \phi(t) \in L^{2}\left(\mathscr{R}_{+}\right)\right\}, \quad E=\left\{g: g(t)=e^{\lambda t} / \phi(t), \lambda \in H\right\}$ and $\alpha(\phi)=$ $\sup \{\operatorname{Re} \lambda: \lambda \in H\}$ where $\alpha(\phi)=-\infty$ if $H$ is empty. $G$ will denote the infinitesimal generator of the semigroup $\left\{S_{t}\right\}$.

2. Basic facts about $\mathscr{A}$. In this section we shall show that each $A_{f}$ is a bounded linear operator on $L^{2}\left(\mathscr{R}_{+}\right)$, that the mapping $f \rightarrow A_{f}$ of $L^{2}\left(\mathscr{R}_{+}\right)$onto $\mathscr{A}_{0}$ is a continuous linear mapping, that $\mathscr{A}_{0}$ is an algebra, and that $\mathscr{A}$ is a maximal abelian algebra.

Lemma 2.1. $\left\|A_{f}\right\| \leqq \rho\|f\|$ for all $f$ in $L^{2}\left(\mathscr{R}_{+}\right)$where

$$
\rho=\sup _{x} \int_{0}^{x}\left(\frac{\phi(x)}{\phi(t) \phi(x-t)}\right)^{2} d t
$$

Proof. Let $g \in L^{2}\left(\mathscr{R}_{+}\right)$. To see that $A_{f}$ is well-defined we note that

$$
\begin{aligned}
\left(A_{f} g\right)(x) & =\int_{0}^{\infty} \frac{f(t)}{\phi(t)}\left(S_{t} g\right)(x) d t \\
& =\int_{0}^{x} \frac{\phi(x)}{\phi(t) \phi(x-t)} f(t) g(x-t) d t
\end{aligned}
$$

and the integral exists since $f$ and $g$ are square integrable and $\phi$ is continuous and nonzero. We note further that 


$$
\left|\left(A_{f} g\right)(x)\right|^{2} \leqq \int_{0}^{x}\left(\frac{\phi(x)}{\phi(t) \phi(x-t)}\right)^{2} d t \int_{f}^{x}|f(t) g(x-t)|^{2} d t
$$

Therefore

$$
\left\|A_{f} g\right\|^{2} \leqq \rho^{2} \int_{0}^{\infty} \int_{0}^{x}|f(t) g(x-t)|^{2} d t d x=\rho^{2}\|f\|^{2}\|g\|^{2}
$$

so that $A_{f}$ is a bounded linear operator on $L^{2}\left(\mathscr{R}_{+}\right)$and $\left\|A_{f}\right\| \leqq \rho\|f\|$.

LeMma 2.2. (i) $A_{\alpha f+\beta g}=\alpha A_{f}+\beta A_{g}$,

(ii) $A_{f g} g=A_{g} f$, and

(iii) $A_{f} A_{g}=A_{A_{g} g}$

for all $f$ and $g$ in $L^{2}\left(\mathscr{R}_{+}\right)$and all complex numbers $\alpha$ and $\beta$.

Proof. (i) and (ii) follow immediately from equation (2). To prove (iii) we let $f, g, h \in L^{2}\left(\mathscr{R}_{+}\right)$and note that

$$
\begin{aligned}
\left(A_{f} A_{g} h\right)(x)=\int_{t=0}^{x} \frac{\phi(x)}{\phi(t) \phi(x-t)} f(t)\left(A_{g} h\right)(x-t) d t \\
=\int_{t=0}^{x} \frac{\phi(x)}{\phi(t) \phi(x-t)} f(t) \int_{s=0}^{x-t} \frac{g(s) \phi(x-t)}{\phi(s) \phi(x-t-s)} h(x-t-s) d s d t \\
=\int_{t=0}^{x} \frac{\phi(x)}{\phi(t) \phi(x-t)} f(t) \int_{s=t}^{x} \frac{g(s-t) \phi(x-t)}{\phi(s-t) \phi(x-s)} h(x-s) d s d t \\
=\int_{s=0}^{x}\left[\int_{t=0}^{s} \frac{\phi(x)}{\phi(t) \phi(s-t) \phi(x-s)} f(t) g(s-t) h(x-s) d t\right] d s \\
=\int_{s=0}^{x} \frac{\phi(x)}{\phi(s) \phi(x-s)}\left[\int_{t=0}^{s} \frac{\phi(s)}{\phi(t) \phi(s-t)} f(t) g(s-t) d t\right] h(x-s) d s \\
=\int_{s=0}^{x} \frac{\phi(x)}{\phi(s) \phi(x-s)}\left(A_{f} g\right)(s) h(x-s) d s \\
=\left(A_{A_{g} g} h\right)(x)
\end{aligned}
$$

Thus (iii) holds for all $f$ and $g$.

It now follows immediately from Lemma 2.2 that $\mathscr{A}_{0}$ is a commutative algebra and from Lemma 2.1 that the mapping $f \rightarrow A_{f}$ is continuous. An easy computation shows that this mapping is one-toone. We state these results in the following theorem. 
THEOREM 2.3. $\mathscr{A}_{0}$ is a commutative algebra of operators on $L^{2}\left(\mathscr{R}_{+}\right)$ and the mapping $f \rightarrow A_{f}$ is a continuous, one-to-one, linear mapping of $L^{2}\left(\mathscr{R}_{+}\right)$onto $\mathscr{A}_{0}$.

It follows from the Open Mapping Theorem and Theorem 2.3 that $\mathscr{A}_{0}$ is closed in the uniform topology if and only if the mapping $f \rightarrow \mathscr{A}_{f}$ is bicontinuous. It is an open question whether or not $\mathscr{A}_{0}$ is closed in the uniform operator topology.

LEMMA 2.4. If $T$ is an operator on $L^{2}\left(\mathscr{R}_{+}\right)$which is in the commutant of $\mathscr{A}_{0}$, then $T A_{f}=A_{T f}$ for each $f$ in $L^{2}\left(\mathscr{R}_{+}\right)$.

Proof. Let $f$ and $g$ be elements of $L^{2}\left(\mathscr{R}_{+}\right)$. Then $T A_{f} g=T A_{g} f=$ $A_{g} T f=A_{T f}$. Consequently $T A_{f}=A_{T f}$ as desired.

Lemma 2.5. $\left\{S_{i}\right\} \subset \mathscr{A}-\mathscr{A}_{0}$.

Proof. Let $f_{n}=n \phi \psi[r, r+1 / n]$ where $\psi[a, b]$ is the characteristic function of $[a, b]$. Then $f_{n} \in L^{2}\left(\mathscr{R}_{+}\right)$and $A_{f_{n}}=n \int_{r}^{r+1 / n} S_{t} d t$ which, because of the strong continuity of $S_{t}$, converges strongly to $S_{r}$. Consequently $S_{r} \in \mathscr{A}$. To see that $S_{r} \notin \mathscr{A}_{0}$ we assume the contrary: $S_{r}=\int_{0}^{\infty}(f(t) / \phi(t)) S_{t} d t$ for some $f$ in $L^{2}\left(\mathscr{R}_{+}\right)$. Consequently, $S_{r}^{*}(g / \phi)=$

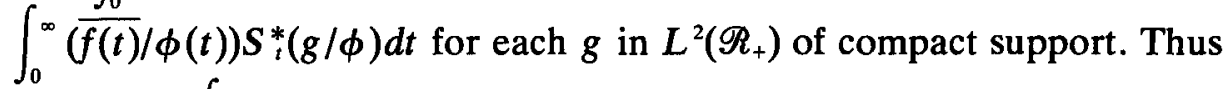
$g(x+r)=\int_{0}^{\infty}(\overline{f(t)} / \phi(t)) g(x+t) d t$. If we define $K(y, s)=$

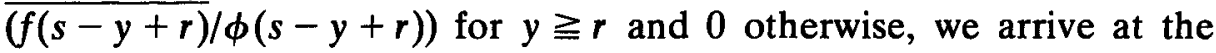
integral equation $g(y)=\int_{0}^{\infty} K(y, s) g(s) d s$. Since the identity is not an integral operator on $L^{2}\left(\mathscr{R}_{+}\right)[5$, p. 87], we arrive at a contradiction and our proof is complete.

An immediate consequence of Lemma 2.5 is that the weakly closed algebra $\mathscr{A}_{1}$ generated by $\left\{S_{t}\right\}$ is a subalgebra of $\mathscr{A}$. Since each element of $\mathscr{A}_{0}$ is obviously in $\mathscr{A}_{1}$, we see that $\mathscr{A}_{1}=\mathscr{A}$; that is, the weakly closed algebra generated by the semigroup $\left\{S_{t}\right\}$ is the same as the weakly closed algebra generated by $\left\{\boldsymbol{A}_{f}\right\}$.

THEOREM 2.6. $\mathscr{A}$ is a maximal abelain algebra and $\mathscr{A}_{0}$ is a proper ideal of $\mathscr{A}$.

Proof. That $\mathscr{A}$ is abelian follows from the fact that $\mathscr{A}_{0}$ is 
abelian. Thus by Lemma 2.4 if $T \in \mathscr{A}$, then $T A_{f}=A_{T f} \in \mathscr{A}_{0}$, proving that $\mathscr{A}_{0}$ is an ideal of $\mathscr{A}$. Lemma 2.5 assures us that $\mathscr{A}_{0}$ is proper and that $I \in \mathscr{A}$. Choose a net $g_{\lambda}$ such that $A_{g_{\lambda}}$ converges weakly to the identity operator $I$. Then since $T A_{g_{\lambda}}=A_{T g_{\lambda}}$, we have $T=\lim A_{T_{g \lambda}}$ and hence $T \in \mathscr{A}$, proving that each element of the commutant of $\mathscr{A}$ is an element of $\mathscr{A}$. The proof that $\mathscr{A}$ is maximal abelian is complete.

We observe that no $A_{f}$ is invertible since $\mathscr{A}_{0}$ is a proper ideal of the maximal abelian algebra $\mathscr{A}$. We shall study in more detail the spectral properties of elements of $\mathscr{A}_{0}$ in the next section.

3. Spectral properties of $\mathscr{A}$. In this section we first characterize certain multiplicative linear functionals on $\mathscr{A}$ and then use this information to study the spectra of elements of $\mathscr{A}$. In particular we are able to show in Corollary 3.4 that whenever $g_{\lambda} \in L^{2}\left(\mathscr{R}_{+}\right)$where $g_{\lambda}(x)=$ $e^{\lambda x} / \phi(x)$, then $g$ is an eigenvector for each element of $\mathscr{A}^{*}$. For an element $A_{f}$ of $\mathscr{A}_{0}$ we then show in Theorem 3.5 that the eigenvalues corresponding to the $g_{\lambda}$ together with the real number 0 make up the entire spectrum of $A_{f}^{*}$.

THEOREM 3.1. If $m$ is a multiplicative linear functional on $\mathscr{A}$, then there exists a unique $g$ in $L^{2}\left(\mathscr{R}_{+}\right)$such that

(i) $m\left(A_{f}\right)=\langle f, g\rangle$ and

(ii) $A_{f}^{*}=\langle g, f\rangle g$ for all $f$ in $L^{2}\left(\mathscr{R}_{+}\right)$.

Conversely, if $m$ and $g$ satisfy (i) and (ii) and $g \neq 0$, then

(iii) $A^{*} g=\left(\langle g, A g\rangle /\|g\|^{2}\right) g$ for all $A$ in $\mathscr{A}$

and $m$ can be extended to a multiplicative linear functional $K$ on $\mathscr{A}$ such that

(iv) $K(A)=\langle A g, g\rangle /\|g\|^{2}$ for all $A$ in $\mathscr{A}$.

Proof. Assume that $m$ is a multiplicative linear functional on $\mathscr{A}$ and define $L(f)=m\left(A_{f}\right)$ for each $f$ in $L^{2}\left(\mathscr{R}_{+}\right)$. It follows from Theorem 2.3 that $L$ is a continuous linear functional on $L^{2}\left(\mathscr{R}_{+}\right)$. By the Riesz Representation Theorem there exists a unique element $g$ of $L^{2}\left(\mathscr{R}_{+}\right)$such that $L(f)=\langle f, g\rangle$ for all $f$. Consequently $m\left(A_{f}\right)=\langle f, g\rangle$ for all $f$ in $L^{2}\left(\mathscr{R}_{+}\right)$.

Assuming now that $m$ is multiplicative, we have for all $f$ and $h$ in $L^{2}\left(\mathscr{R}_{+}\right)\langle h,\langle g, f\rangle g\rangle=\langle f, g\rangle\langle h, g\rangle=m\left(A_{f}\right) m\left(A_{h}\right)=m\left(A_{f} A_{h}\right)=m\left(A_{A_{f} h}\right)=$ $\left\langle A_{f} h, g\right\rangle=\left\langle h, A_{f}^{*} g\right\rangle$. Consequently $A_{f}^{*} g=\langle g, f\rangle g$ as desired.

Assume now that $m$ and $g$ satisfy (i) and (ii) and that $g \neq 0$. Reversing the computation in the preceding paragraph, we conclude that $m$ is a multiplicative linear functional on $\mathscr{A}_{0}$. We shall construct a multiplicative linear extension of $m$ on $\mathscr{A}_{0}$. Let $A \in \mathscr{A}$ 
and $A_{f_{\lambda}} \rightarrow A$ weakly. Then $\langle g, A g\rangle=\lim \left\langle g, A_{f_{\lambda}} g\right\rangle=\lim \left\langle A_{f_{\lambda}}^{*} g, g\right\rangle=$ $\lim \left\langle g, f_{\lambda}\right\rangle\|g\|^{2}$ by (ii). Consequently $\lim \left\langle g, f_{\lambda}\right\rangle=\langle g, A g\rangle /\|g\|^{2}$ and for each $h$ in $L^{2}\left(\mathscr{R}_{+}\right),\langle g, A h\rangle=\lim \left\langle g, A_{f_{A}} h\right\rangle=\lim \left\langle\left\langle g, f_{\lambda}\right\rangle g, h\right\rangle=$ $\langle g, h\rangle\langle g, A g\rangle /\|g\|^{2}$. Thus $A^{*} g=\left(\langle g, A g\rangle /\|g\|^{2}\right) g$, proving the final assertion. We now define $K(A)=\left(\langle A g, g\rangle /\|g\|^{2}\right)$ for each $A$ in $\mathscr{A}$. A straightforward computation shows that $K$ is a multiplicative linear functional on $\mathscr{A}$ and that $K$ is an extension of $m$.

We have shown that to each multiplicative linear functional on $\mathscr{A}$ there corresponds a unique element $g$ of $L^{2}\left(\mathscr{R}_{+}\right)$which is a common eigenvector for the elements of $\mathscr{A}^{*}$, provided $g \neq 0$. In Theorem 3.3 we shall show that each such function $g$ is necessarily of the form $e^{\lambda t} / \phi(t)$ for some complex number $\lambda$.

LEMMA 3.2. If $G$ is the generator of $\left\{S_{t}\right\}$ and $\lambda$ is sufficiently large, then $A_{f}=(\lambda-G)^{-1}$ where $f(t)=e^{-\lambda t} \phi(t)$.

Proof. Since $\left\{S_{t}\right\}$ is strongly continuous, there exist constants $M$ and $w$ so that $\sup _{x}|\phi(x+t) / \phi(x)|=\left\|S_{t}\right\| \leqq M e^{w t}[2$, Lemma 2.1]. Thus $\phi(t) \leqq M e^{w t}$ and for $\lambda$ sufficiently large $f(t)=e^{-\lambda t} \phi(t) \in L^{2}\left(\mathscr{R}_{+}\right)$. Then $A_{f}=\int_{0}^{\infty}(f(t) / \phi(t)) S_{t} d t=\int_{0}^{\infty} e^{-\lambda t} S_{t} d t=(\lambda-G)^{-1}$. [6, p. 344].

THEOREM 3.3. If $m$ is a multiplicative linear functional on $\mathscr{A}$ and $g$ satisfies

(i) $m\left(A_{f}\right)=\langle f, g\rangle$ and

(ii) $A_{f}^{*} g=\langle g, f\rangle g$ for all $f$ in $L^{2}\left(\mathscr{R}_{+}\right)$, then either $g=0$ or there exists a complex number $\lambda$ such that $g(x)=$ $e^{\lambda x} / \phi(x)$. Conversely, if $g(x)=e^{\lambda x} / \phi(x)$ and $g \in L^{2}\left(\mathscr{R}_{+}\right)$, then $g$ satisfies (ii).

Proof. Let $g$ satisfy (i) and (ii). By Lemma $3.2\left(\lambda^{*}-G^{*}\right)^{-1} g=$ $\langle g, f\rangle g$ where $f(t)=e^{-\lambda t} \phi(t) \in L^{2}\left(\mathscr{R}_{+}\right)$. If $\langle g, f\rangle=0$, then $g=0$. Assume that $\langle g, f\rangle \neq 0$. Since $A_{f}^{*} g=\langle g, f\rangle g$, we have

$$
\langle g, f\rangle g(x)=\frac{e^{\lambda x}}{\phi(x)} \int_{x}^{\infty} \frac{\phi(t)}{e^{\lambda t}} g(t) d t . \quad \text { a.e. }
$$

Let $h(x)=\phi(x) g(x) / e^{\lambda x}$ and note that $h \in L^{1}\left(\mathscr{R}_{+}\right)$since $\phi(x) / e^{\lambda x} \in$ $L^{2}\left(\mathscr{R}_{+}\right)$and $g \in L^{2}\left(\mathscr{R}_{+}\right)$. We now have $\langle g, f\rangle h(x)=\int_{x}^{\infty} h(t) d t$ a.e. Since $h$ is integrable and $\langle g, f\rangle \neq 0$, we can conclude first that $h$ is continuous and secondly that $h$ is differentiable. Thus $\langle g, f\rangle h^{\prime}(x)=$ 
$-h(x)$ and $h(x)=A e^{\beta x}$ or equivalently $g(x)=A e^{(\lambda+\beta) x} / \phi(x)$. It follows from

(3) that $\langle g, f\rangle g(0)=(1 / \phi(0)) \int_{0}^{\infty}\left(\phi(t) g(t) / e^{\lambda t}\right) d t=$ $(1 / \phi(0))\langle g, f\rangle$. Thus $g(0)=1 / \phi(0)$, so that $A=1$ and $g(x)=e^{(\lambda+\beta) x} / \phi(x)$, as desired. A straightforward computation shows that if $g$ is of this form, then $g$ satisfies (ii).

As an immediate consequence of Theorems 3.3 and 3.1 we have:

Corollary 3.4. If $g_{\lambda}(t)=e^{\lambda t} / \phi(t) \in L^{2}\left(\mathscr{R}_{+}\right)$, then $A^{*} g_{\lambda}=$ $\left(\left\langle g_{\lambda}, A g_{\lambda}\right\rangle /\left\|g_{\lambda}\right\|^{2}\right) g_{\lambda}$ for all $A$ in $\mathscr{A}$.

In the remainder of the paper we let $H=\left\{\lambda: e^{\lambda t} / \phi(t) \in L^{2}\left(\mathscr{R}_{+}\right)\right\}$and $E=\left\{g: g(t)=e^{\lambda t} / \phi(t), \lambda \in H\right\}$. We shall show that both sets are either empty or large: more precisely, either $H$ is empty or $H$ is a closed half-plane and at the same time either $E$ is empty or its linear span is dense in $L^{2}\left(\mathscr{R}_{+}\right)$.

THEOREM 3.5. $\sigma\left(A_{f}\right)=\{\langle f, g\rangle: g \in E\} \cup\{0\}$.

Proof. In our comments following Theorem 2.6 we observed that $0 \in \sigma_{\mathscr{A}}\left(A_{f}\right)$ whenever $f \in L^{2}\left(\mathscr{R}_{+}\right)$. By Theorem 2.6 $\mathscr{A}$ is a maximal abelian algebra and hence for each $A$ in $\mathscr{A}, \sigma(A)=\sigma_{\mathscr{A}}(A)=\{m(A): m$ a multiplicative linear functional on $\mathscr{A}\}$. By Theorems 3.1 and 3.3, $m\left(A_{f}\right)=\langle f, g\rangle$ for some $g$ in $E$ provided $m$ is not identically zero on $\mathscr{A}_{0}$, completing the proof.

We observed in the proof of Theorem 3.5 that $\sigma(A)=\{m(A): m$ a multiplicative linear functional on $\mathscr{A}\}$ which implies that $\sigma(A) \supset$ $\left\{\langle A g, g\rangle /\|g\|^{2}: g \in E\right\} \cup\left\{m_{0}(A)\right\}$ where $m_{0}$ is identically zero on $\mathscr{A}_{0}$. It is not known whether this set is the entire spectrum of $A$; equivalently, it is not known if $m_{0}$ is unique.

Corollary 3.6. Among the conditions

(i) $\mathscr{A}$ contains a nonzero quasinilpotent element;

(ii) $\sigma\left((\beta-G)^{-1}\right)=\{0\}$ for some $\beta$ such that $(\beta-G)^{-1}$ is bounded ;

(iii) $\sigma\left(A_{f}\right)=\{0\}$ for all $f$ in $L^{2}\left(\mathscr{R}_{+}\right)$;

(iv) $E=\varnothing$;

(v) the linear span of $E$ is not dense in $L^{2}\left(\mathscr{R}_{+}\right)$, the following implications hold: (i) $\Rightarrow$ (ii) $\Leftrightarrow$ (iii) $\Leftrightarrow$ (iv) $\Leftrightarrow$ (v).

Proof. (i) $\Rightarrow$ (v). If $\sigma(A)=\{0\}$, then by Theorem 3.1(iii) $A^{*} g=0$ for each $g$ in $E$. Thus if $A$ is nonzero, the linear span of $E$ is not dense 
in $L^{2}\left(\mathscr{R}_{+}\right)$and (v) holds. (v) $\Rightarrow$ (ii). If $\sigma\left((\beta-G)^{-1}\right) \neq\{0\}$ for sufficiently large $\beta$, then by Theorem 3.5 there exist $g(t)=e^{\lambda t} / \phi(t) \in E$. If the linear span of $E$ is not dense in $L^{2}\left(\mathscr{R}_{+}\right)$, then there exists a nonzero $f$ such that

$$
\begin{aligned}
0 & =\int \frac{e^{z t}}{\phi(t)} f(t) d t \text { whenever } \operatorname{Re} z \leqq \operatorname{Re} \lambda \\
& =\int e^{(z-\lambda) t} \frac{e^{\lambda t} f(t)}{\phi(t)} d t \\
& =\int e^{x x t} \frac{e^{\lambda t} f(t)}{\phi(t)} d t \text { for } z=\lambda+i x, x \text { real. }
\end{aligned}
$$

Thus the Fourier coefficients of the $L^{1}\left(\mathscr{R}_{+}\right)$function $e^{\lambda t} f(t) / \phi(t)$ are zero, implying that $f=0$ a.e. This contradiction completes the proof that (v) $\Rightarrow$ (ii). (ii) $\Rightarrow$ (iii) $\Rightarrow$ (iv) by Theorem 3.5; (iv) $\Rightarrow$ (v) trivially.

The following two examples demonstrate the two different types of symbols $\phi$ : in the first example $\alpha(\phi)>-\infty$ and $H$ is a half plane and in the second example $\alpha(\phi)=-\infty$ and $H$ is empty. Thus in the second example each $A_{f}$ is quasinilpotent.

EXAmple 1. Let $\phi(x)=x+1$. We shall show that $\phi$ is of bounded kernel type and $\alpha(\phi)=0$.

$$
\int_{0}^{x} \frac{\phi(x)^{2}}{\phi(t)^{2} \phi(x-t)^{2}} d t=2(x+1)^{2}\left[\frac{\log (x+1)}{(x+3)^{3}}+\frac{x}{(x+2)^{2}(x+1)}\right]
$$

which is bounded on $\mathscr{R}_{+}$. To see that $\alpha(\phi)=0$ we note that $\int_{0}^{\infty}\left|e^{\lambda x} /(x+1)\right|^{2} d x$ converges for $\operatorname{Re} \lambda \leqq 0$ and diverges for $\operatorname{Re} \lambda>$ 0 . Thus by Corollary 3.6 no element of $\mathscr{A}$ is quasinilpotent and by Corollary $3.4 \mathrm{~g}$ is a common eigenvector for $A^{*}$ whenever $g(x)=$ $e^{\lambda x} / \phi(x), \operatorname{Re} \lambda \leqq 0$.

EXAMPLE 2. Let $\phi(x)=e^{-x^{2} / 2}$. We shall show that $\phi$ is of bounded kernel type and $\alpha(\phi)=-\infty$. Obviously for each complex number $\lambda e^{\lambda t} / \phi(t) \notin L^{2}$ so that $\alpha(\phi)=-\infty$. To see that $\phi$ is of bounded kernel type we compute as follows

$$
\int_{0}^{x}\left(\frac{\phi(x)}{\phi(t) \phi(x-t)}\right)^{2} d t=\int_{0}^{x} \frac{e^{-x^{2}}}{e^{-t^{2}} e^{-(x-t)^{2}}} d t
$$




$$
\begin{aligned}
& =\int_{0}^{x} e^{-2 t(x-t)} d t \\
& =e^{-x^{2} / 2} \int_{0}^{x} e^{(x-2 t)^{2} / 2} d t \\
& =e^{-x^{2} / 2} \int_{-x}^{x} \frac{1}{2} e^{\frac{1}{s^{2}}} d s \\
& =e^{-x^{2} / 2} \int_{0}^{x} e^{\frac{1}{2} s^{2}} d s .
\end{aligned}
$$

Thus by l'Hopital's Rule

$$
\lim _{x \rightarrow \infty} \int_{0}^{x}\left(\frac{\phi(x)}{\phi(t) \phi(x-t)}\right)^{2} d x=\lim _{x \rightarrow \infty} \frac{\frac{d}{d x} \int_{0}^{x} e^{\frac{1}{s^{2}} d x}}{\frac{d}{d x} e^{x^{2} / 2}}=0 .
$$

Consequently since $\int_{0}^{x}(\phi(x) / \phi(t) \phi(x-t))^{2} d x$ is continuous and vanishes at $\infty$, it is bounded.

We note also for this example that since $\left\|S_{t}^{n}\right\|^{1 / n}=e^{-n t^{2} / 2}$, each $S_{t}(t \neq 0)$ is quasinilpotent. The fact that each $A_{f}$ is quasinilpotent follows from Corollary 3.6.

Although it appears difficult in general to determine which symbols $\phi$ are of bounded kernel type, in certain cases one can use information about the set $H$ to show that $\phi$ is not of bounded kernel type. More precisely, if $\phi$ is of bounded kernel type, then $H$ is a closed half plane. To see this we argue as follows. Assume $\lambda \in H$. Then $\int_{0}^{\infty}\left(e^{2 \operatorname{Re} \lambda x} / \phi(x)^{2}\right) d x=\int_{0}^{\infty}\left|e^{\lambda x} / \phi(x)\right|^{2} d x<\infty$. Consequently if $\operatorname{Re} z \leqq$ $\operatorname{Re} \lambda$, then $z \in H$, proving that $H$ is a half plane. Now choose $\beta$ so that $f(t)=e^{-\beta t} \phi(t) \in L^{2}\left(\mathscr{R}_{+}\right)$and $(\beta-G)^{-1}$ is bounded. By Lemma 3.2 and Theorem $3.5 \sigma\left((\beta-G)^{-1}\right)=\{1 /(\beta-\lambda): \lambda \in H\} \cup\{0\}$. Thus $\{1 /(\beta-\lambda): \lambda \in H\} \cup\{0\}$ is compact and it easily follows that $H$ is closed.

In [3] it was shown that $\phi(x)=(x+1)^{-1 / 2}$ is the symbol for a subnormal weighted translation semigroup. Since $\int_{0}^{\infty}\left|e^{\lambda t} / \phi(t)\right|^{2} d t$ converges for $\operatorname{Re} \lambda<0$ and diverges otherwise we see that $H$ is not closed and hence $\phi$ is not of bounded kernel type. At the end of $\$ 5$ we shall see that no subnormal weighted translation semigroup has symbol of bounded kernel type. Indeed a stronger conclusion is obtained: if $\left\{S_{t}\right\}$ is hyponormal $\left(S_{t}^{*} S_{t} \gg S_{t} S_{t}^{*}\right.$ for each $\left.t\right)$, then the symbol $\phi$ of $\left\{S_{t}\right\}$ is not of bounded kernel type. 
4. Transitivity. For clarity in this section we' shall let $\mathscr{A}_{\phi}$ denote the weakly closed algebra generated by $\left\{S_{t}\right\}$, where $\phi$ is the symbol of $\left\{S_{t}\right\}$ and $\phi$ is of bounded kernel type.

Let $T$ be a linear transformation with domain $D(T) \subseteq X$. We say that $T$ commutes with $A$ in $B(X)$ if $A D(T) \subseteq D(T)$ and $A T x=T A x$ for each $x$ in $D(T)$. Also, $T$ commutes with a set of operators $S$ if it commutes with each operator in $S . \quad T$ is said to be closable if $T$ has a closed extension.

THEOREM 4.1. If $T$ is a densely defined linear transformation commuting with $\mathscr{A}_{\phi}$, then $T$ is closable and $T A_{h}$ is bounded for every $h$ in $D(T)$.

Proof. To prove that $T$ is closable we must show that if $\left\{h_{n}\right\}$ is a sequence in $D(T)$ converging to 0 and $\left\{T h_{n}\right\}$ converges to some vector $f$, then $f=0$. Note that if $u$ is in $D(T)$ and $v$ is in $L^{2}\left(\mathscr{R}_{+}\right)$, then $A_{u} v=A_{v} u$ is in $D(T)$. Let $g$ be in $D(T)$. Then

$$
T A_{h_{n}} g=A_{h_{n}} T g \rightarrow 0
$$

But $T A_{h n} g=T A_{g} h_{n}=A_{g} T h_{n} \rightarrow A_{g} f$. Thus for every $g$ in $D(T), A_{g} f=$ 0 . But since $D(T)$ is dense in $L^{2}\left(\mathscr{R}_{+}\right),\left\{A_{g}: g\right.$ in $\left.D(T)\right\}$ is weakly dense in $\mathscr{A}_{\phi}$. Since $I$ is in $\mathscr{A}_{\phi}$, we have $f=0$. Hence $T$ is closable.

Now suppose $h$ is in $D(T)$. Since $T A_{h}$ commutes with $\mathscr{A}_{\phi}, T A_{h}$ is closable. But $T A_{h}$ is everywhere defined, so $T A_{h}$ is in $B\left(L^{2}\right)$. In fact, since $T A_{h} f=T A_{f} h=A_{f} T h=A_{T h} f$, we have $T A_{h}=A_{T h}$.

Note that with $T$ as in the above theorem and $h$ in $D(T),\left(T A_{h}\right)^{*}$ is, of course, bounded. Explicitly, $\left(T A_{h}\right)^{*}=T^{*} A_{h}^{*}$. To see this let $f$ also be in $D(T)$ and let $g$ be in $L^{2}\left(\mathscr{R}_{+}\right)$. Then

$$
\begin{aligned}
\left\langle T f, A_{h}^{*} g\right\rangle & =\left\langle A_{h} T f, g\right\rangle \\
& =\left\langle T A_{h} f, g\right\rangle \\
& =\left\langle f,\left(T A_{h}\right)^{*} g\right\rangle
\end{aligned}
$$

so that $A_{h}^{*} g$ is in $D\left(T^{*}\right)$ and $\left(T A_{h}\right)^{*} g=T^{*} A_{h}^{*} g$.

The properties of transformations commuting with the algebra $\mathscr{A}_{\phi}$ just developed are nicely applicable to the theory of transitive algebras. An algebra $\mathscr{T}$ of operators on $X$ is transitive if the only closed subspaces of $X$ invariant under all the operators in $\mathscr{T}$ are $\{0\}$ and $X$. For general discussions of transitive algebras see [1] and [7, Chapter 8]. The following result is an immediate corollary to Arveson's density theorem.

Proposition. (Arveson). If $\mathscr{T}$ is a transitive algebra with the 
property that every linear transformation commuting with $\mathscr{T}$ is a multiple of the identity, then $\mathscr{T}$ is weakly dense in $B(X)$.

Now if $T$ is a closed densely defined linear transformation commuting with the transitive algebra $\mathscr{T}$ and either $T$ or $T^{*}$ has an eigenvector (other than 0 ), then $T$ is a multiple of the identity. Since $T^{*}$ commutes with $\mathscr{T}^{*}=\left\{A^{*}: A\right.$ in $\left.\mathscr{T}\right\}$ and $\mathscr{T}^{*}$ is transitive if and only if $\mathscr{T}$ is, it suffices to justify the above remark in the case $T x=\lambda x, x \neq 0$. But then for every $A$ in $\mathscr{T}, T A x=A T x=\lambda A x$ so $T-\lambda I=0$ on $\{A x: A$ in $\mathscr{T}\}$ which is dense in $X$. But one sees easily that a closed transformation agreeing with a bounded operator on a dense set is in fact that bounded operator, and so $T=\lambda I$.

We now apply these remarks to certain algebras of the form $\mathscr{A}_{\phi}$. Recall that $\alpha(\phi)=\sup \left\{\lambda\right.$ in $\left.\mathscr{R}: \int_{0}^{\infty}\left(e^{2 \lambda x} / \phi^{2}(x)\right) d x<\infty\right\}$.

THEOREM 4.2. If $\phi$ is of bounded kernel type and $\alpha(\phi)>-\infty$, then every transitive algebra containing $\mathscr{A}_{\phi}$ is weakly dense in $B\left(L^{2}\right)$.

Proof. We have seen that every densely defined linear transformation commuting with $\mathscr{A}_{\phi}$ is closable. It is easy to show that the minimal closed extension of a closable transformation $L$ commutes with all the operators commuting with $L$. Let $T$ be a closed linear transformation commuting with $\mathscr{A}_{\phi}$. Then for each $h$ in $D(T)$, we have seen that $T^{*} A_{h}^{*}$ is in $\mathscr{A}_{\phi}^{*}$. Let $g(x)=e^{\alpha(\phi) x} / \phi(x)$. Then $g$ is in $L^{2}\left(\mathscr{R}_{+}\right)$and $A_{f}^{*} g=$ $(g, f) g$ for each $f$ in $L^{2}\left(\mathscr{R}_{+}\right)$. Now $T^{*} A_{h}^{*}=\left(T A_{h}\right)^{*}=A_{T h}^{*}$, so

$$
\langle g, T h\rangle g=T^{*} A_{h}^{*} g=\langle g, h\rangle T^{*} g
$$

Thus $g$ is an eigenvector for $T^{*}((g, h)$ cannot be 0 for all $h$ in the dense set $D(T)$ ). It follows from the proposition preceding this theorem that every transitive algebra containing $\mathscr{A}_{\phi}$ is dense.

Question. What about transitivity considerations in the case $\alpha(\phi)=-\infty$ ?

5. Functional properties of $\phi$. We now concentrate on some properties of the function $\phi \rightarrow \alpha(\phi)$. Throughout the following discussion we assume that $\phi$ is in $C^{1}([a, \infty))$ for some $a \geqq 0$ and that $\phi(x) \neq 0$ for all $x \geqq 0$. Note that Theorem 5.2 is not dependent upon $\phi$ being of bounded kernel type. Define 


$$
\begin{aligned}
& i(\phi)=\liminf _{t \rightarrow \infty} \frac{\phi^{\prime}(t)}{\phi(t)} \\
& s(\phi)=\limsup _{t \rightarrow \infty} \frac{\phi^{\prime}(t)}{\phi(t)} .
\end{aligned}
$$

LEMMA 5.1. If $\phi$ is of bounded kernel type, then $\alpha(\phi)<\infty$.

Proof. We have seen that for $\operatorname{Re} \lambda \leqq \alpha(\phi) g_{\lambda}(x)=e^{\lambda x} / \phi(x)$ is in $L^{2}\left(\mathscr{R}_{+}\right)$and for every $f$ in $L^{2}\left(\mathscr{R}_{+}\right)\left(f, g_{\bar{\lambda}}\right)=\int_{0}^{\infty}\left(e^{\lambda x} / \phi(x)\right) f(x) d x$ which is the Laplace transform of $f / \phi, L f$, evaluated at $-\lambda$. Thus if $\alpha(\phi)=\infty, L f$ is entire for each $f$ in $L^{2}\left(\mathscr{R}_{+}\right)$. But $\left\langle f, g_{\bar{\lambda}}\right\rangle$ is in the spectrum of $A_{f}$ so $L f$ is bounded and entire. By Liouville's Theorem $L f$ is constant for every $f$ in $L^{2}$. But then $f / \phi=0$ and $f=0$. Thus $\alpha(\phi)<\infty$.

THEOREM 5.2. If $\phi$ is in $C^{1}[a, \infty)$ for some $a \geqq 0$ and $\phi(x) \neq 0$ for all $x$ then $i(\phi) \leqq \alpha(\phi) \leqq s(\phi)$.

Proof. We prove only the inequality $i(\phi) \leqq \alpha(\phi)$, the other inequality's validity being quite similarly (and symmetrically) ascertained. If $i(\phi)=-\infty$ the inequality holds. Assume first that $i(\phi)$ is finite. Let $\epsilon>0$ and let $\lambda=i(\phi)-\epsilon$. Then since $\phi^{\prime} / \phi$ is continuous for $t \geqq a$ we have $\phi^{\prime}(t) / \phi(t)>\lambda+(\epsilon / 2)$ for all $t \geqq a$. Let $f(x)=$ $e^{2 \lambda x} / \phi^{2}(x)$. Then $f^{\prime}(x) / f(x)=2\left(\lambda-\left(\phi^{\prime}(x) / \phi(x)\right)\right)<-\epsilon$ for all $x \geqq a$ hence $f(x) \leqq f(a) e^{-\varepsilon(x-a)}$ for all $x \geqq a$. Since $f$ is continuous on $[0, a], f$ is in $L^{1}\left(\mathscr{R}_{+}\right)$. Thus $i(\phi)-\epsilon \leqq \alpha(\phi)$ for all $\epsilon>0$ and so $i(\phi) \leqq \alpha(\phi)$.

Now, if $i(\phi)=+\infty$ then we have $\lim _{t \rightarrow \infty}\left(\phi^{\prime}(t) / \phi(t)\right)=+\infty$. But then we easily see that for any $\lambda$ in $\mathscr{R}$ the function $f$ defined above is in $L^{\prime}$ and so $\alpha(\phi)=+\infty$.

CoROllaRY 5.3. If $\lim _{t \rightarrow \infty}\left(\phi^{\prime}(t) / \phi(t)\right)$ exists, then $\alpha(\phi)=$ $\lim _{t \rightarrow \infty}\left(\phi^{\prime}(t) / \phi(t)\right)$.

In order to see that strict inequalities in the above Theorem 5.2 are possible, even for $\phi$ of bounded kernel type, note that if $h$ and $1 / h$ are bounded continuous functions on $\mathscr{R}_{+}$and $\phi$ is of bounded kernel type, then $h \phi$ also is of bounded kernel type. Moreover, one easily verifies that $\alpha(\phi)=\alpha(h \phi)$. However (assuming $h$ is in $C^{1}([a, \infty))$ ) for $\rho=h \phi$, $\rho^{\prime} / \rho=\left(h^{\prime} / h\right)+\left(\phi^{\prime} / \phi\right)$. If, for example, we let $\phi(x)=x+1$ and $h(x)=$ $2+\sin x$ then all requirements above are satisfied, $\lim _{t \rightarrow \infty}\left(\phi^{\prime}(t) / \phi(t)\right)=0$, $\liminf _{t \rightarrow \infty}\left(h^{\prime}(t) / h(t)\right)=-\sqrt{3} / 3, \quad \lim \sup _{t \rightarrow \infty}\left(h^{\prime}(t) / h(t)\right)=\sqrt{3} / 3$, and so $i(\rho)=-\sqrt{3} / 3, \alpha(\rho)=0$, and $s(\rho)=\sqrt{3} / 3$.

We conclude the paper by showing that the class of weighted 
translation semigroups with symbol of bounded kernel type is disjoint from a rather large class of weighted translation semigroups, including the hyponormal (and of course subnormal) ones.

LEMMA 5.4. For $\phi$ of bounded kernel type in $C^{1}([a, \infty))$ for some $a>0, \alpha(\phi)<s=\sup _{t \geqq a}\left(\phi^{\prime}(t) / \phi(t)\right)$.

Proof. We have already seen that $\alpha(\phi)<\infty$ so the case $s=\infty$ is obvious. Suppose then that $s<\infty$. Then for $t \geqq a, \phi^{\prime}(t) / \phi(t) \leqq s$ so $\phi(t) / \phi(a) \leqq e^{s(t-a)}$ and hence $1 / \phi^{2}(t) \geqq\left(1 / \phi^{2}(a)\right) e^{2 s(a-t)}$. We then have

$$
\infty>\int_{0}^{\infty} \frac{e^{2 \alpha(\phi) t}}{\phi^{2}(t)} d t \geqq \int_{0}^{a} \frac{e^{2 \alpha(\phi) t}}{\phi^{2}(t)} d t+\left(\int_{a}^{\infty} e^{2[\alpha(\phi)-s] t} d t\right) e^{2 s a}
$$

Thus $\alpha(\phi)<s$, for otherwise the last integral diverges.

COROllary 5.5. If $\left\{S_{t}\right\}$ is a hyponormal weighted translation semigroup with symbol $\phi$ in $C^{1}([a, \infty))$ for some $a \geqq 0$, then $\phi$ is not of bounded kernel type.

Proof. In [2] we showed that $\left\{S_{t}\right\}$ is hyponormal if and only if $\log \phi$ is convex. Thus $\phi^{\prime} / \phi$ is an increasing function and so $\lim _{t \rightarrow \infty}\left(\phi^{\prime}(t) / \phi(t)\right)=\sup _{t \geq 0}\left(\phi^{\prime}(t) / \phi(t)\right)$. By Corollary 5.3 and Lemma 5.4 $\phi$ cannot be of bounded kernel type.

Note that if $\left\{S_{t}\right\}$ is subnormal, the condition of $\phi$ being in $C^{1}([a, \infty))$ holds automatically since $\phi$ has the form $\phi^{2}(x)=e^{\alpha x} \int_{0}^{\infty} e^{-t x} d \rho(t)$ where $\rho$ is a probability measure.

\section{REFERENCES}

1. W. B. Arveson, A density theorem for operator algebras, Duke Math. J., 34 (1967), 634-647.

2. M. R. Embry and A. Lambert, Weighted translation semigroups, to appear in Rocky Mountain Math. J.

3. - Subnormal weighted translation semigroups, J. Functional Anal., 24, No. 3 (1977), 268-275.

4. Richard Frankfurt, Quasicyclic subnormal semigroups, to appear in Canad. J. Math.

5. P. R. Halmos, A Hilbert Space Problem Book, Van Nostrand, Princeton, N. J., 1967.

6. E. Hille and R. S. Phillips, Functional analysis and semigroups, Amer. Math. Soc., Providence,

R. I., 1957.

7. H. Radjavi and P. Rosenthal, Invariant Subspaces, Springer-Verlag, New York, N. Y. 1973.

Received April 20, 1977 and in revised form June 2, 1977.

UNIVERSITY OF NoRTH CAROlina

ChARLotTE, NC 28223 



\section{PACIFIC JOURNAL OF MATHEMATICS}

\section{EDITORS}

RICHARD ARENS (Managing Editor)

University of California

Los Angeles, CA 90024

R. A. BeAumont

University of Washington

Seattle, WA 98105

C. C. MOORE

University of California

Berkeley, CA 94720
J. DUGUNDJI

Department of Mathematics

University of Southern California

Los Angeles, CA 90007

R. FINN AND J. MILGRAM

Stanford University

Stanford, CA 94305

\section{ASSOCIATE EDITORS}
E. F. BECKENBACH
B. H. NEUMANN
F. WOLF
K. YoshidA

\section{SUPPORTING INSTITUTIONS}

UNIVERSITY OF BRITISH COLUMBIA

UNIVERSITY OF SOUTHERN CALIFORNIA

CALIFORNIA INSTITUTE OF TECHNOLOGY

STANFORD UNIVERSITY

UNIVERSITY OF CALIFORNIA

UNIVERSITY OF HAWAII

MONTANA STATE UNIVERSITY

UNIVERSITY OF TOKYO

UNIVERSITY OF NEVADA

UNIVERSITY OF UTAH

NEW MEXICO STATE UNIVERSITY

OREGON STATE UNIVERSITY

UNIVERSITY OF OREGON

OSAKA UNIVERSITY

WASHINGTON STATE UNIVERSITY

UNIVERSITY OF WASHINGTON

AMERICAN MATHEMATICAL SOCIETY

The Supporting Institutions listed above contribute to the cost of publication of this Journal, but they are not owners or publishers and have no responsibility for its contents or policies.

Mathematical papers intended for publication in the Pacific Journal of Mathematics should be in typed form or offset-reproduced (not dittoed), double spaced with large margins. Underline Greek letters in red, German in green, and script in blue. The first paragraph or two must be capable of being used separately as a synopsis of the entire paper. Items of the bibliography should not be cited there unless absolutely necessary, in which case they must be identified by author and Journal, rather than by item number. Manuscripts, in duplicate, may be sent to any one of the four editors. Please classify according to the scheme of Math. Reviews, Index to Vol. 39. All other communications should be addressed to the managing editor, or Elaine Barth, University of California, Los Angeles, California, 90024.

100 reprints are provided free for each article, only if page charges have been substantially paid. Additional copies may be obtained at cost in multiples of 50 .

The Pacific Journal of Mathematics is issued monthly as of January 1966. Regular subscription rate: $\$ 72.00$ a year (6 Vols., 12 issues). Special rate: $\$ 36.00$ a year to individual members of supporting institutions.

Subscriptions, orders for numbers issued in the last three calendar years, and changes of address should be sent to Pacific Journal of Mathematics, 103 Highland Boulevard, Berkeley, California, 94708.

PUBLISHED BY PACIFIC JOURNAL OF MATHEMATICS, A NON-PROFIT CORPORATION

Printed at Jerusalem Academic Press, POB 2390, Jerusalem, Israel. 


\section{Pacific Journal of Mathematics}

\section{Vol. 75, No. $2 \quad$ October, 1978}

Susan Jane Zimmerman Andima and W. J. Thron, Order-induced

topological properties ................................... 297

Gregory Wade Bell, Cohomology of degree 1 and 2 of the Suzuki groups . . 319

Richard Body and Roy Rene Douglas, Rational homotopy and unique

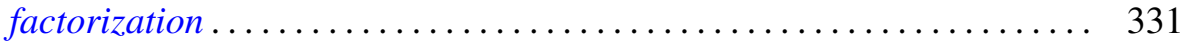

Frank Lewis Capobianco, Fixed sets of involutions ................. 339

L. Carlitz, Some theorems on generalized Dedekind-Rademacher sums .... 347

Mary Rodriguez Embry and Alan Leslie Lambert, The structure of a special class of weighted translation semigroups .....................

Steve Ferry, Strongly regular mappings with compact ANR fibers are

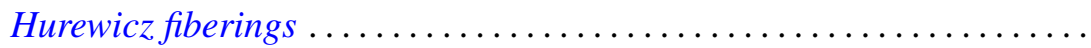

Ivan Filippenko and Marvin David Marcus, On the unitary invariance of the

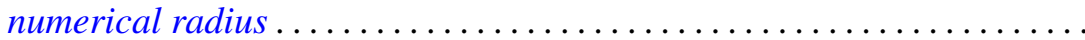

$\mathrm{H}$. Groemer, On the extension of additive functionals on classes of convex

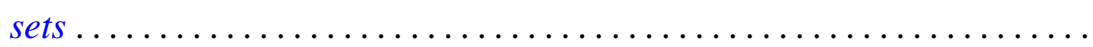

Rita Hall, On the cohomology of Kuga's fiber variety ............... 411

H. B. Hamilton, Congruences on $\mathrm{N}$-semigroups ................. 423

Manfred Herrmann and Rolf Schmidt, Regular sequences and lifting

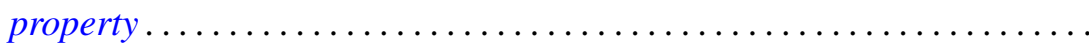

James Edgar Keesling, Decompositions of the Stone-Čech compactification which are shape equivalences .....................

Michael Jay Klass and Lawrence Edward Myers, On stopping rules and the expected supremum of $S_{n} / T_{n}$

Ronald Charles Linton, $\lambda$-large subgroups of $C_{\lambda}$-groups

William Owen Murray, IV and L. Bruce Treybig, Triangulations with the free cell property ............................

Louis Jackson Ratliff, Jr., Polynomial rings and $H_{i}$-local rings ...

Michael Rich, On alternate rings and their attached Jordan rings....

Gary Sampson and H. Tuy, Fourier transforms and their Lipschitz

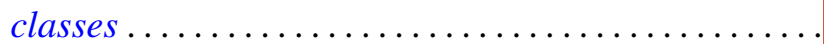

Helga Schirmer, Effluent and noneffluent fixed points on dendrites ...

Daniel Byron Shapiro, Intersections of the space of skew-symmetric maps

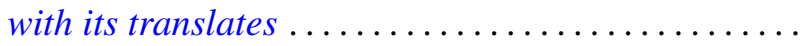

Edwin Spanier, Tautness for Alexander-Spanier cohomology ...

Alan Stein and Ivan Ernest Stux, A mean value theorem for binary digits ...

Franklin D. Tall, Normal subspaces of the density topology . .

William Yslas Vélez, Prime ideal decomposition in $F\left(\mu^{1 / p}\right) \ldots$ 